\title{
Probing Spintronic Features in Conduction through Silicene and Graphene Barriers
}

\author{
DIANA MENESES-GUSTIN ${ }^{1}$ and MARCOS R.S. TAVARES ${ }^{1,2}$ \\ 1.-Centro de Ciências Naturais e Humanas, Universidade Federal do ABC, Santo André, \\ SP 09210-170, Brazil. 2.—e-mail: marcos.tavares@ufabc.edu.br
}

\begin{abstract}
The Dirac electrons in silicene experience stronger spin-orbit interaction (SOI) than in graphene due to silicene's band buckled two-dimensional (2D) structure. In this work we theoretically probe the main effects of the SOI in silicene, provided this interaction can be controlled by an external electrical field. Attention is paid to how silicene's SOI effects can turn into graphene's once external parameters can be regulated. By comparing the electronic transmission through silicene and graphene structures we are able to fit the external electrical field to obtain similar results for both materials. We study the conductance through silicene barriers and also show how to straightforwardly probe spin polarization and spin-resolved transmission using as few parameters as possible. We first calculate the electronic transmission through single and double barriers as a function of the electron's angle of incidence $\theta$, the electron energy $E$, and the strength of the external electrical field $E_{z}$. We then found that the polarization $P$ versus $\theta$ in double-barrier structures exhibits quasi-periodic resonances. We finally study asymmetric structures that allow the presence of more transmission channels in the conductance.
\end{abstract}

Key words: Silicene, graphene, spintronics, electronics

\section{INTRODUCTION}

Within the physics of two-dimensional (2D) materials, silicene has attracted a great deal of attention, in the first place, because it presents Dirac cones as an electronic structure that is similar to graphene's. Silicene is a monolayer honeycomb structure of silicon and has been synthesized in laboratory recently. ${ }^{1-3}$ In fact, the previous cornerstone achievement of the carbon-based graphene opened up a channel to investigate different materials with similar 2D properties. Among many candidates, silicon-based material gets the most attention provided the spread of silicon usage in actual (and foremost) electronic devices.

On the contrary to graphene, in which the spinorbit interaction (SOI) is depreciable, silicene has a strong SOI that leads to an energy gap of $1.55 \mathrm{meV}$. Such a gap exists due to the buckled $2 \mathrm{D}$ structure of

(Received December 16, 2017; accepted July 10, 2018; published online July 30,2018 ) silicene. This structure results from the large ionic radius of silicon. It can be controlled by an external electric field $E_{z}$, which turn out to be an important parameter in studying spin and valley polarization in graphene. These interesting features led to seminal studies on specific points regarding silicene, such as the spin-Hall effect, ${ }^{4}$ the anomalous Hall effect, ${ }^{5,6}$ the capacitance of an electrically tunable silicene device, ${ }^{7}$ and others. ${ }^{8}$

It is worth mentioning that there is another $2 \mathrm{D}$ material, phosphorene, which has also been synthesized recently. ${ }^{9-12}$ This material has an inherent, direct and appreciable band gap that depends on the number of its layers. It serves as a field effect transistor and is more stable than silicene, but it loses stability when it is grown upon substrates that destroy the Dirac cones. By this token, we point out that substrate effects can be mimicked by the SOI parameter in $2 \mathrm{D}$ materials ${ }^{13}$ so that in the present paper we intend to deal with a timely issue indeed.

Since the SOI can also lead to spin-resolved transport, pertinent also to quantum computing 
and other important magnetic effects, it is worth further studying SOI in silicene and contrast these results to graphene's ${ }^{13-15}$ and even to phosphorene. We then carefully study here the electronic transmission, polarization and conductance through single and double potential barriers in silicene. These quantities are shown to be very sensitive to parameters such as the electron's angle of incidence $\theta$, the electron energy $E$, and the strength of the external field $E_{z}$. We believe these results provide important insights regarding electronic transport in silicene. It was suggested that $E_{z}$ alone might not be able to lift spin resolution in silicene due to valley-to-valley symmetry, and, therefore, an external magnetic field should be present in order to locally break this time-reversal symmetry ${ }^{8,16}$ and reveal polarization. However, to avoid including further external physical parameters such as the magnetic field (both its orientation and intensity), which surely brings extra physical nuances to the calculation, especially into the valley-to-valley symmetry properties, we choose to look at one valley only and simply probe the eventual spin polarization that would certainly be enhanced in the presence of further external fields. In this way, we present a simpler (but scholarly sound) way to probe the spin resolution, which is, by virtue of the SOI, an inherent feature of these materials. For completeness sake, we also calculate the spin-resolved conductance for the double barrier case. This conductance presents many resonance features, which can be compared to measurements in laboratory. We also present these conductances for different barrier and well parameters and show how sensitive these results can be. Once we discussed the transmission coefficient, important emphasis is given to the conductivity between asymmetric barrier structures.

The manuscript is organized as follows. The section "Theoretical Formalism" is devoted to define the used formulation. In "Single Versus Double Barriers" and "Conductance for Symmetric and Asymmetric Structures" sections explore, respectively, the pertinent cases of single and double barrier structures. We conclude our work in the "Conclusions".

\section{THEORETICAL FORMALISM}

The single-particle Hamiltonian for carriers in a single $K$ valley in silicene is given by ${ }^{4}$

$$
\begin{aligned}
H_{K} & =\left(\begin{array}{ll}
H_{++} & H_{+-} \\
H_{-+} & H_{--}
\end{array}\right) \\
= & \left(\begin{array}{cccc}
s_{-} & \hbar v_{F} k_{-} & i a \lambda_{R 2} k_{-} & 0 \\
\hbar v_{F} k_{+} & -s_{-} & -i \lambda_{R 1} & -i a \lambda_{R 2} k_{+} \\
-i a \lambda_{R 2} k_{+} & i \lambda_{R 1} & -s_{+} & \hbar v_{F} k_{-} \\
0 & i a \lambda_{R 2} k_{+} & \hbar v_{F} k_{+} & s_{+}
\end{array}\right),
\end{aligned}
$$

$$
s_{ \pm}=\lambda_{S O I} \pm \ell E_{z}
$$

Here $2 \ell$ is the distance between the two sublattices $A$ and $B, v_{F}$ the Fermi velocity, $a=0.386 \mathrm{~nm}$ the lattice constant, and the wave number $k_{ \pm}=k_{x} \pm i k_{y}$. Also, for the diagonal elements of Eq. $1, \lambda_{\text {SOI }} \equiv \lambda \simeq 3.9 \mathrm{meV}$ is the strength of the SOI in the lattice, and $E_{z}$ is the external electric field. But it has been noted that the secondary (Rashba) spin coupling parameters $\lambda_{R 1}=10 \mu \mathrm{eV}$ and $\lambda_{R 2}=0.7 \mathrm{meV}$ are indeed too small in comparison to the primary one $\lambda_{\mathrm{SOI}}$. If we consider that, Eq. 1 should easily turn into two decoupled $2 \times 2$ matrices. That is what we are going to do in first place. So, we hereafter deliberately assume $H_{-+}=H_{+-}$ $\simeq 0$ in Eq. 1

The Rashba spin-couplings (RSCOs) are responsible for breaking the sub-lattice symmetry of the system's honeycomb structure while the intrinsic spin orbit coupling (ISOC), $\lambda_{S O I}$, gives rise to the spin-split band gap. In fact, the single-valley (flavor) Dirac physics, determined by two independent Dirac-like cones at $K$ and $K^{\prime}$ points, dominates as long as there is no valley mixing, which is the most probably regime if $\lambda_{S O I}$ is much greater than $\lambda_{R 1}$ and $\lambda_{R 2}$. By tuning $E_{z}$, one should be able to study a critical value at which the system, at the topological insulating phase, goes into a semi-metal state, which is reasonably protected as valley-spinlocked-metal (VSLM) from the opposite spin-
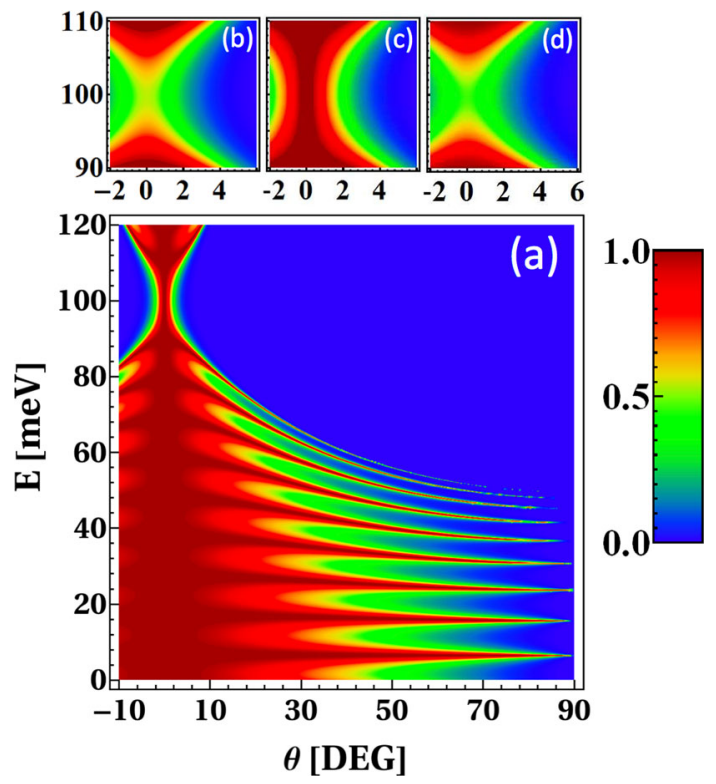

Fig. 1. (Color online) (a) $(E, \theta)$ contour plot of the transmission through a single barrier for spin-up electrons in silicene. The width and height of the barrier are $W=110 \mathrm{~nm}$ and $U=100 \mathrm{meV}$, respectively, the field $E_{z}=170 \mathrm{meV} / \mathrm{nm}$, and $v_{F}=5.5 \times 10^{5} \mathrm{~m} \mathrm{~s}$. Panels (b), (c), and (d) show the region $(E, \theta)$ delimited by $-2 \leq \theta \leq 6$ and $90 \leq E \leq 110 \mathrm{meV}$ and correspond, respectively, to $E_{z}=50,170$, and $300 \mathrm{meV} \mathrm{nm}$. Red (blue) represents $100 \%(0 \%)$ chance of transmission.

where 
polarization of the $K$ or $K^{\prime}$ valley. The crossover to VSLM is referred to as the topological phase transition (TPT). Upon further increasing $E_{z}$, the system may naturally turn into a insulator. The Klein tunneling is a good way to settle this issue experimentally because the Dirac electrons have the total transmission probability (TTP), since there is one for the normal incidence on a two-dimensional barrier (a silicene based N-P-N nano-transistor). On the contrary, the Dirac electrons through such a barrier are expected to show TTP less than one. At the TPT, therefore, one should expect a sudden upswing in the total transmission probability. This is what we are going to check out in this paper.

Therefore, in the following we are going to vary the value of $E_{z}$ and study such effects as TTP and TPT taking the advantage from the very fact that $\lambda_{\text {SOI }}$ is much greater than $\lambda_{R 1}$ and $\lambda_{R 2}$, so one can consider just one valley flavor without any significant loss of completeness in the discussion of the results. The resulting $K$-flavor matrix is then blockdiagonal with its upper (lower) block pertaining to spin-up (spin-down) electrons alone.

The spin-down Hamiltonian is given by

$$
H_{--}=\left(\begin{array}{cc}
-\lambda-\ell E_{z} & \hbar v_{F} k_{-} \\
\hbar v_{F} k_{+} & \lambda+\ell E_{z}
\end{array}\right)=\left(\begin{array}{cc}
-s_{+} & \hbar v_{F} k_{-} \\
\hbar v_{F} k_{+} & s_{+}
\end{array}\right)
$$

where the Hamiltonian for spin-up electrons $H_{++}$is easily given by the same Eq. 3, but with $s_{+}$replaced by $-s_{-}$. The eigenvectors of (3) are straightforwardly written in the usual form of two-component spinors, i.e., $\Psi=\left(\varphi_{A}^{\downarrow}, \varphi_{B}^{\downarrow}\right)^{T}$ for spin-down and $\Psi=\left(\varphi_{A}^{\uparrow}, \varphi_{B}^{\uparrow}\right)^{T}$ for spin-up electrons with $A$ and $B$ labelling the two sublattices and $T$ denoting the transpose. With $\mu= \pm$, the corresponding eigenvalues are

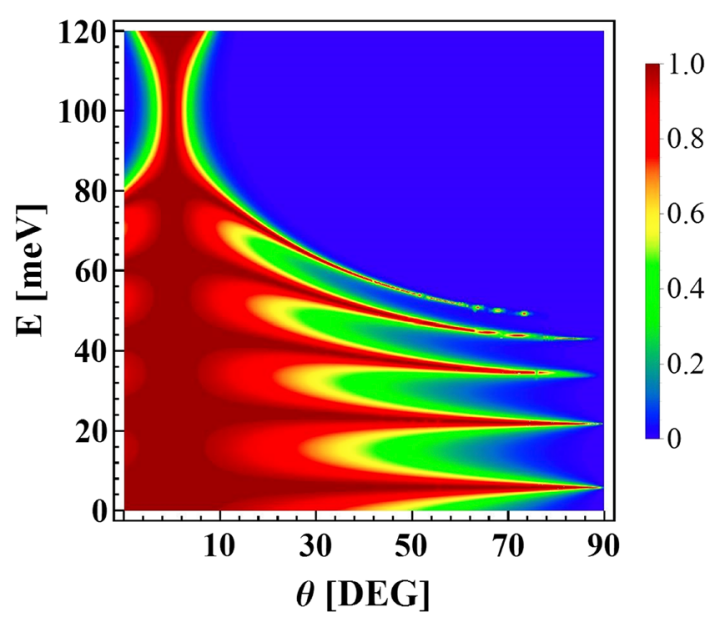

Fig. 2. (Color online) $(E, \theta)$ contour plot of the transmission through a single barrier for graphene, where $v_{F}=10^{6} \mathrm{meV}$. The width and height of the barrier are $W=110 \mathrm{~nm}$ and $U=100 \mathrm{meV}$, respectively. Red (blue) represents $100 \%(0 \%)$ chance of transmission.

$$
E_{k \mu}=\mu\left[\hbar^{2} v_{F}^{2} k^{2}+s_{ \pm}^{2}\right]^{1 / 2} .
$$

We leave the detailed forms of eigenvectors of (3) for the next sections where we study the single and double barriers structures.
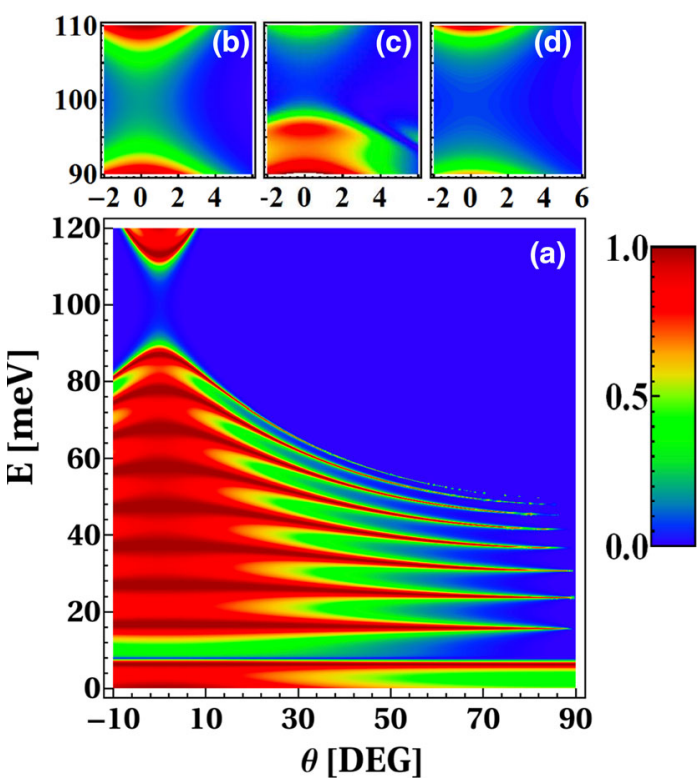

Fig. 3. (a) $(E, \theta)$ contour plot of the transmission through a single barrier for spin-down electrons in silicene. The width and height of the barrier are $W=110 \mathrm{~nm}$ and $U=100 \mathrm{meV}$, respectively, the field $E_{z}=170 \mathrm{meV} / \mathrm{nm}$, and $v_{F}=5.5 \times 10^{5} \mathrm{~m} / \mathrm{s}$. Panels (b), (c), and (d) show the region $(E, \theta)$ delimited by $-2 \leq \theta \leq 6$ and $90 \leq E \leq 110$ $\mathrm{meV}$ and correspond, respectively, to $E_{z}=50,170$, and $300 \mathrm{meV} /$ $\mathrm{nm}$. Red (blue) represents $100 \%(0 \%)$ chance of transmission

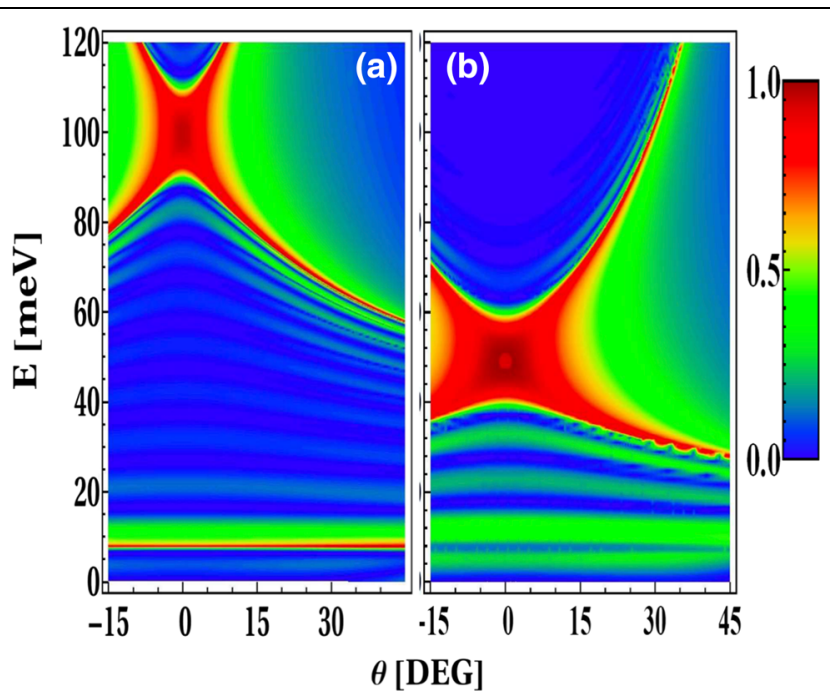

Fig. 4. (Color online) (a) Contour plot of the polarization for a single barrier in silicene of width and height given by $W=110 \mathrm{~nm}$ and $U=100 \mathrm{meV}$, respectively, the field $E_{z}=170 \mathrm{meV} / \mathrm{nm}$, and $v_{F}=5.5 \times 10^{5} \mathrm{~m} / \mathrm{s}$. (b) The same as in part (a), but with $U=50$ meV. Red (blue) represents $100 \%(0 \%)$ chance of transmission. 


\section{SINGLE VERSUS DOUBLE BARRIERS}

In this section we first consider a single barrier of a constant height $U$ and of width $W$ along the $x$ axis and infinitely long along the $y$ axis. Such a constant potential $U(x)$ is then included into the singleparticle Hamiltonian (3). Along the $y$-direction, the eigenvectors are simple plane waves $e^{i k_{y} y}$, once the electrons are free from any force there. The same happens for the $x$-direction to the left (region I) and to the right (region II) of the barrier. So, for region I, the eigenvectors are written in the form of plane wave

$$
\varphi_{A}^{I}=e^{i \eta x}+r e^{-i \eta x}
$$

where $r$ is the reflection amplitude. For region III, it is in the form

$$
\varphi_{A}^{I I I}=t e^{i \eta x}
$$

with $t$ the transmission amplitude. In the barrier (region II), the eigenvector is written as

$$
\varphi_{A}^{I I}=a e^{i \delta x}+b e^{-i \delta x} .
$$

Here the parameters $\delta^{2}=\left[(U-E)^{2}-s_{ \pm}^{2}\right] / \hbar^{2} v_{F}^{2}-k_{y}^{2}$, and $\eta^{2}=\left(E^{2}-s_{ \pm}^{2}\right) / \hbar^{2} v_{F}^{2}-k_{y}^{2}$, where $v_{F}$ is the Fermi velocity. We mention, concerning the component $\varphi_{B}$, that through the diagonalization of Eq. 3, one can easily find

$$
\varphi_{B}=i \hbar v_{F}\left(\partial \varphi_{A} / \partial x-k_{y} \varphi_{A}\right) /\left(s_{ \pm}+U-E\right),
$$

from which the component $\varphi_{B}$ can be written for the three barrier regions.

The electronic transmission $T$ is obtained after matching $\varphi_{A}$ and $\varphi_{B}$ at the interfaces between regions I and II and regions II and III. Within such

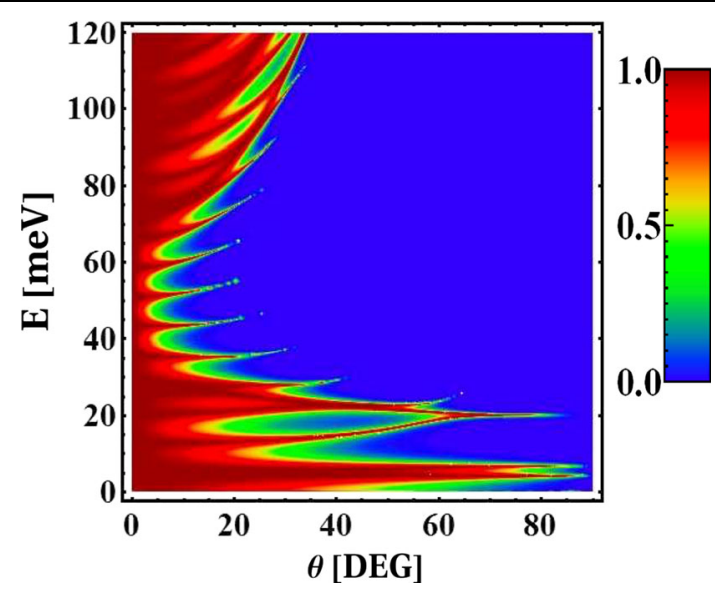

Fig. 5. (Color online) $(E, \theta)$ contour plot of the transmission through a double barrier for spin-up electrons in silicene. The width and height of the barriers are $W=50 \mathrm{~nm}$ and $U=50 \mathrm{meV}$, respectively, the inter-barrier separation $d=100 \mathrm{~nm}$, and the field $E_{z}=170 \mathrm{meV} /$ $\mathrm{nm}$. Red (blue) represents $100 \%(0 \%)$ chance of transmission. a procedure, the transmission $T=t t^{*}$ takes that standard form obtained whenever the Dirac-like Hamiltonian is used instead of Schrödinger's equation in calculating the transmission $T$. After a straightforward calculation, the matching conditions lead us to the transmission coefficient for spinup (+) and spin-down (-) electrons written as

$$
T_{ \pm}=1 /\left[1+F \sin ^{2}(W \delta)\right],
$$

with $F=b_{-} b_{+} /\left[2 \eta \delta\left(s_{ \pm}-E\right)\left(s_{ \pm}+U-E\right)\right]^{2} \quad$ and $b_{ \pm}=\left[\eta\left(s_{ \pm}+U-E\right) \pm \delta\left(s_{ \pm}-E\right)\right]^{2}+v_{F}^{2} k_{y}^{2}$. As in the graphene case, ${ }^{13,14}$ we see $T_{ \pm}$with clear dependence on $k_{y}$, which is absent $\left(k_{y}=0\right)$ in usual semiconductor barriers. But in contrast, now $T$ depends on $s_{ \pm}$as well, and, for $s_{ \pm}=0$, Eq. 9 gives the graphene result, naturally, from which one analytically obtains $T=1$ for normal incidence $\left(k_{y}=0\right)$. So, the electrons are totally transmitted when they are injected in a normal direction with respect to the barrier.

In Fig. 1a we show a $(E, \theta)$ contour plot of the transmission $T_{\uparrow}$ for spin-up electrons for a single barrier in silicene. The width and height of the barrier are $W=110 \mathrm{~nm}$ and $U=100 \mathrm{meV}$, respectively. Intentionally, we set $E_{z}=170 \mathrm{meV} / \mathrm{nm}$ because this value of $E_{z}$, together with $\ell=0.23 \AA$ and $\lambda_{S O I}=3.9 \mathrm{meV}$, gives $s_{-}=0$ and makes the diagonal terms in Eq. 1 vanish. In this case we should obtain the graphene results for $v_{F}=10^{6} \mathrm{~m} / \mathrm{s}$. This is indeed the case as shown in Fig. 2, which represents the same $(E, \theta)$ contour plot for graphene. Figure 2 differs very slightly from Fig. 1a because we used $v_{F}=5.5 \times 10^{5} \mathrm{~m} / \mathrm{s}$, which is appropriate for silicene. Notice that, as more tunneling channels open up for silicene, the red neck of the resembled skeleton gets a little narrower for $E \simeq U$ when compared to graphene's. This reflects the

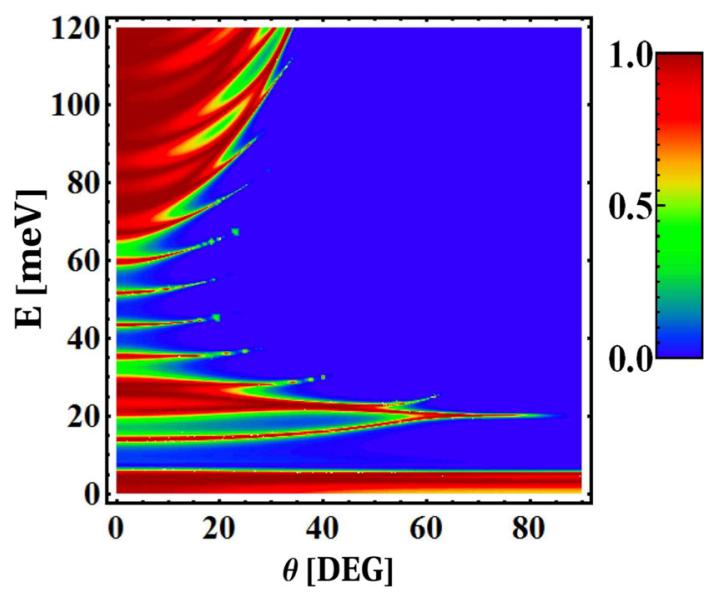

Fig. 6. (Color online) $(E, \theta)$ contour plot of the transmission through a double barrier for spin-down electrons in silicene. The width and height of the barriers are $W=50 \mathrm{~nm}$ and $U=50 \mathrm{meV}$, respectively, the inter-barrier separation $d=100 \mathrm{~nm}$, and the field $E_{z}=170 \mathrm{meV} /$ $\mathrm{nm}$. Red (blue) represents $100 \%(0 \%)$ chance of transmission. 
lowering of $v_{F}$ and, consequently, the eventual disappearance of the total transmission condition, turning the system back into the usual Schrödinger behavior, in the sense that a natural interference process between the transmitted and reflected Schrödinger wave-function occurs around this energy.

But questions arise here regarding the influence of changing $E_{z}$ on the silicene results. How this external electric field impact on the transmission results and correspondent conductance? In order to completely address that, and in view of comparing Figs. 1a to 2, we focus on (the red-neck region) the region of the $(E, \theta)$ plane approximately delimited by $-2 \leq \theta \leq 6$ and $90 \leq E \leq 110 \mathrm{meV}$, in panels (b), (c), and (d) that correspond, respectively, to $E_{z}=50$ , 170, and $300 \mathrm{meV} / \mathrm{nm}$. As can be seen, for values of $E_{z}$ away from, say, the eventual graphene value $E_{z}=170 \mathrm{meV} / \mathrm{nm}$ the perfect total transmission weakens considerably. We show that one is able to suppress the total transmission in silicene by manipulating $E_{z}$. We notice that when the quantity $s_{-}$is a value between two values, $-1.0 \mathrm{meV}$ and 1.0 $\mathrm{meV}$, one is still able to see total transmission for $\theta \approx 0$. But away from these values, i.e panels (b) and (d), the total transmission is indeed affected. These results show the natural strong relationship between the gap, due to the buckled structure, and external electric field $E_{z}$. We show that the latter can also control the transmission. Some specific values for $s_{-}$, which suppress the total transmission for $\theta \approx 0$ are $2.75 \mathrm{meV}$ and $3.00 \mathrm{meV}$, and those which keep it are $0.68 \mathrm{meV}, 0.0 \mathrm{meV}$ and -0.70 meV.

We now consider spin-down electrons and show in Fig. 3 a the corresponding $(E, \theta)$ contour plot of the transmission. The results are similar to those in Fig. 1a for most angles of incidence $\theta$ except for

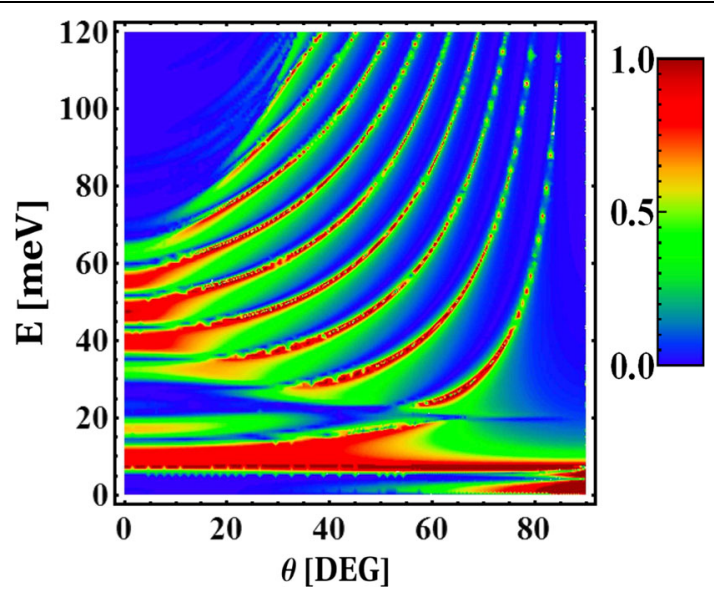

Fig. 7. (Color online) Polarization for a symmetric double barrier in silicene. Here, width and height of the barriers are $W=50 \mathrm{~nm}$ and $U=50 \mathrm{meV}$, respectively, the inter-barrier separation $d=100 \mathrm{~nm}$, and the field $E_{z}=170 \mathrm{meV} / \mathrm{nm}$. Red (blue) represents maximum (minimum) polarization. normal or near-normal incidence, i.e, for $\theta \approx 0$. In this case we have a gap in the transmission approximately for $90 \leq E \leq 110 \mathrm{meV}$ (Eq. 3 for $k_{y}=0$ gives $T=0$ for $\bar{E}=\bar{V} \pm s_{+}$and $E=s_{+}$), i.e., the red neck is now absent. Notice that, together with Fig. 1, one is seeing a spin-resolved transmission. Again, to assess the influence of the field $E_{z}$, we show in panels (b), (c), and (d) a small region containing the total-transmission gap for the same values of $E_{z}$ as in Fig. 1. As can be seen, the main effect of increasing $E_{z}$ away from the value $E_{z}=170$ $\mathrm{meV} / \mathrm{nm}$ is to increase the gap, while for $E_{z}$ less than this value, the gap tends to close. We also notice in passing that, upon reversing the field $E_{z}$, the factor $s_{+}$in Eq. 1 becomes $s_{-}$and the up spins will be blocked, as in Fig. 2, whereas the down spins $\left(s_{-} \rightarrow s_{+}\right)$will be transmitted as in Fig. 1 . This is again a simple demonstration that the $E_{z}$ can be able to probe a very spin-resolved transmission through single barrier.

In order to make the results presented so far increasingly helpful, and to effectively compare the results in Figs. 1a to $3 \mathrm{a}$, we show in Fig. $4 \mathrm{a}$, a $(E, \theta)$ contour plot of the polarization $P$ defined by

$$
P=\left(T_{\uparrow}-T_{\downarrow}\right) /\left(T_{\uparrow}+T_{\downarrow}\right) .
$$

This quantity is defined as the simplest manner to account for the probed spin polarization. The polarization $P$ vanishes in most of the $(\boldsymbol{E}, \theta)$ plane in Fig. 4a. But notice, however, the regions where it doesn't and especially its $100 \%$ value for nearnormal incidence and $90 \leq E \leq 110 \mathrm{meV}$, which corresponds to that of the transmission gap in Fig. 3a. This is drastically different from the case of graphene in which $P$ vanishes everywhere due to the smallness of $\lambda_{\mathrm{SOI}}$ and the vanishing of the distance $\ell$ between the two sublattices.

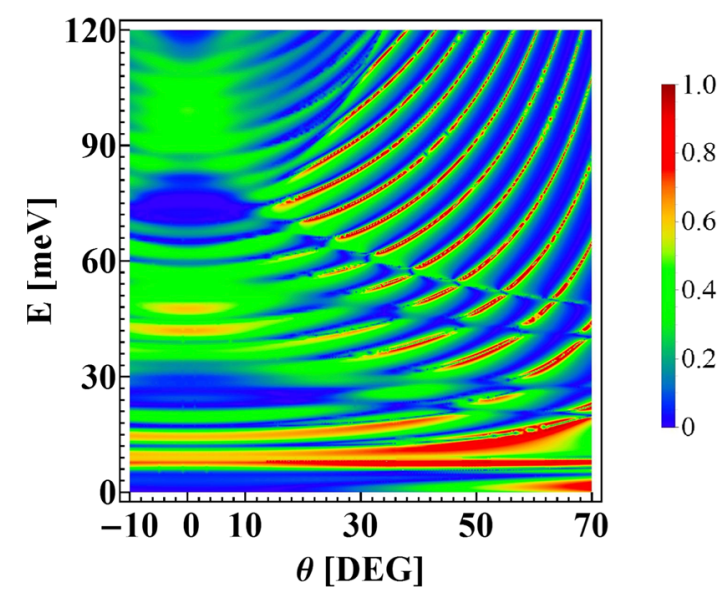

Fig. 8. (Color online) Polarization for a asymmetric double barriers in silicene. The width of the barriers are $W=50 \mathrm{~nm}$, but the first (second) one barrier is of height $U_{1}=100 \mathrm{meV}\left(U_{2}=50 \mathrm{meV}\right)$. The inter-barrier separation is $d=250 \mathrm{~nm}$, and the field $E_{z}=170 \mathrm{meV} /$ $\mathrm{nm}$. Red (blue) represents maximum (minimum) polarization. 


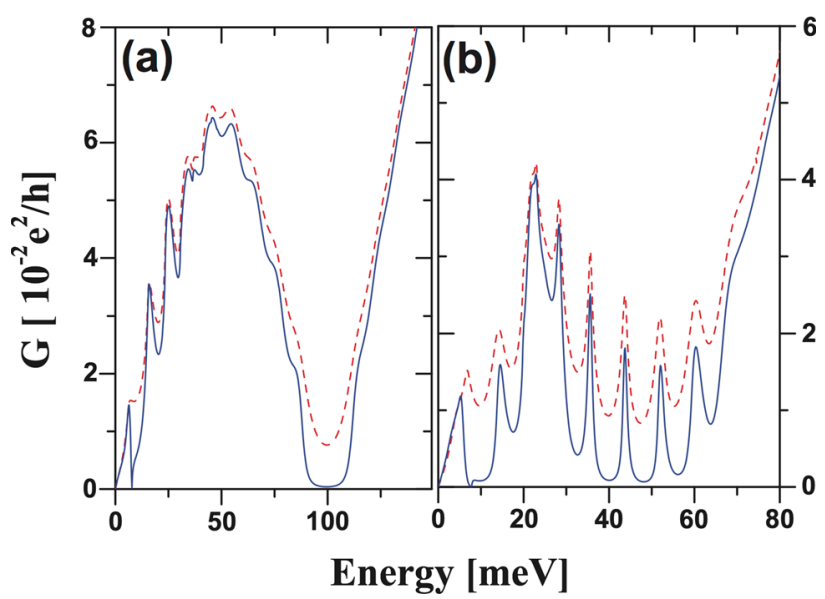

Fig. 9. (Color online) (a) Conductance through a single barrier vs energy $E$ in silicene. The dashed and solid curves are for spin-up $\left(G_{\uparrow}, s_{-}=0\right)$ and spin-down $\left(G_{\downarrow}, s_{+} \neq 0\right)$ electrons, respectively. (b) Conductance through the double barrier described in Fig. 5.

This difference remains as sharp when we reduce the height $U$. The plot in Fig. 4b is drawn for the same values of $W$ and $E_{z}$ as in (a) but for $U=50$ $\mathrm{meV}$. As shown, the most important change is that the red window in (a), centred at $\theta=0, U=100$ $\mathrm{meV}$, is now centred at $\theta=0, U=50 \mathrm{meV}$ and nearly twice as wide. Again, in there $T_{\uparrow}$, controlled by the (graphene) value of $E_{z}$, is almost 1 and $T_{\downarrow} \approx 0$.

For double barriers, the analytic calculation for the transmission, as given in Eq. 9, is more cumbersome (but academically straightforward) and, therefore, for the sake of objectiveness, it will not be written here. We are going to show the numerical results only for this case. We present, then, the $(E, \theta)$ contour for spin-up electrons in Fig. 5 with the double-barrier parameters specified in its caption. Again, for $E_{z}=170 \mathrm{meV} / \mathrm{nm}$ we have the same kind of results seen above (those resembling graphene's) for a $\left(k_{y}, k_{x}\right)$ contour plot, since the diagonal terms in Eq. 1 vanish. But we see many more resonances than in single barriers. By contrasting these results, though, with those in Fig. 6 for spin-down electrons, we see again qualitative differences especially for near-normal incidence with several gaps in the transmission. That is, we have probed a spinresolved transmission that is more complex than that for single barriers. Again, this structure of the transmission is reflected in the $(E, \theta)$ contour plot of the polarization $P$ that we show in Fig. 7. Notice the quasi-periodic character of $P$ versus the angle $\theta$ for energies, say, larger than $80 \mathrm{meV}$. This contour plot contrasts rather sharply with that of Fig. 4 for single barriers as we now see several windows of perfect polarization especially for near-normal incidence.

The results presented so far also raise the question of their sensitivity to the parameters. We emphasize that all contour plots for double barriers are sensitive to their height $U$ and the distance $d$ between them. This is expected since more

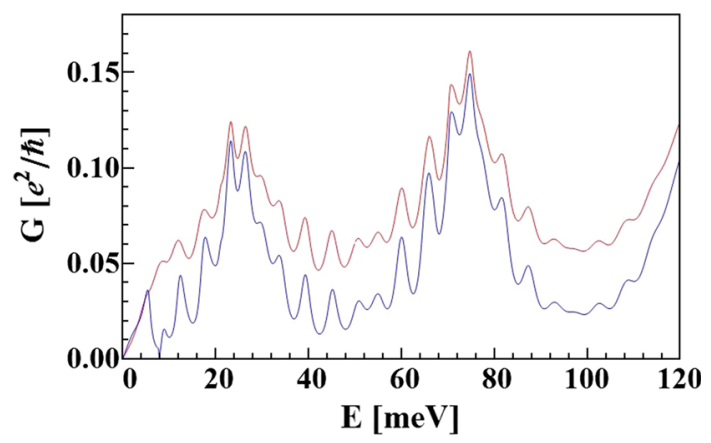

Fig. 10. (Color online) Conductance through an asymmetric silicene structure vs energy $E$. The dashed and solid curves are for spin-up $\left(G_{\uparrow}, s_{-}=0\right)$ and spin-down $\left(G_{\downarrow}, s_{+} \neq 0\right)$ electrons, respectively. The sample parameters are the same as in Fig. 8.

resonances can be created or destroyed by changing $U$ and/or $d$. For the sake of completeness, we show in Fig. 8 the further polarization $(E, \theta)$ contour plot for asymmetric double barriers. Here we increase the height of the first barrier to $100 \mathrm{meV}$, and also increase the distance to $d=250 \mathrm{~nm}$. The remaining parameters are the same as in Fig. 7. Therefore, we reveal the following: (1) an increasing in the transmission channels reflected in the polarization results as the system becomes asymmetric; and (2) a clear memory effect of the bright red region which appears in Fig. 7 around $E=100 \mathrm{meV}$. These effects are absent in symmetric structures.

\section{CONDUCTANCE FOR SYMMETRIC AND ASYMMETRIC STRUCTURES}

We now focus on what may be experimentally seen regarding the previous results. The concern here is the electronic current, which is proportional to a weighted integral of the transmission. ${ }^{17}$ One then imagines to what extension the spin-resolved features survive after such an averaging. We show in Fig. 9 the conductance $G$ using the standard formula ${ }^{18}$

$$
G=\left(e^{2} / \hbar\right) \int T(E, \theta) E \cos \theta d \theta .
$$

The red, dashed curve is for spin-up $\left(G_{\uparrow}, s_{-}=0\right)$ electrons and the blue, solid one for spin-down $\left(G_{\downarrow}, s_{+} \neq 0\right)$ electrons. As shown in panel (a), near the 2nd minimum of the two curves, we have a gap in $G_{\downarrow}$, as well as a dip for $E=10 \mathrm{meV}$, in agreement with Fig. 3. In general, and relative to graphene, the overall silicene results for $G$ are similar, but one sees important qualitative differences as well. This is more pronounced for double barriers for which $G_{\downarrow}$, pertinent to Fig. 6, has four very deep minima or gaps as Fig. 9b demonstrates. All gaps in Fig. 9 could get wider by increasing the value of $E_{z}$, cf. Fig. 3a and d.

For the sake of completeness, we finally show in Fig. 10 the conductances $G_{\uparrow}$ and $G_{\downarrow}$ for the asymmetric structure whose results are shown in Fig 8. The most interesting feature here is that, the more 
asymmetric the structure gets, the more is the number of transmission channels. In particular, the distance $d$ between the barriers plays a important role in getting more channels,but the positions of the two maxima, around the energies $20 \mathrm{meV}$ and $80 \mathrm{meV}$, respectively, do not change appreciably. Therefore, according to our results, the asymmetry between the barriers should increase the number of channels appearing in the conductance.

\section{CONCLUSIONS}

In summary, we carefully evaluated the electronic transmission through single and double barriers in silicene as a function of the angle of incidence $\theta$, the electron energy $E$, and the strength of the field $E_{z}$ and obtained a very reliable probing of spin-resolved transmission, especially for near-normal incidence, and a conductance qualitatively different in some ranges of $E$ than that in graphene. The strength of the resolution depends strongly on the barrier parameters. This is entirely due to the strong SOI in silicene and does not occur in graphene in which the SOI is very weak. We also analyzed the effects of the eventual sample asymmetry between barriers and show that the conductance shape may change appreciably even for small asymmetries.

\section{ACKNOWLEDGEMENTS}

This work was supported by Universidade Federal do ABC and CNPq (Brazil), No. 131134/2012-2014.

\section{REFERENCES}

1. P. Vogt, P. De Padova, C. Quaresima, J. Avila, E. Frantzeskakis, M.C. Asensio, A. Resta, B. Ealet, and G. Le Lay, Phys. Rev. Lett. 108, 155501 (2012).

2. A. Fleurence, R. Friedlein, T. Ozaki, H. Kawai, Y. Wang, and Y. Yamada-Takamura, Phys. Rev. Lett. 108, 245501 (2012).

3. Z. Ni, Q. Liu, K. Tang, J. Zheng, J. Zhou, R. Qin, Z. Gao, D. $\mathrm{Yu}$, and J. Lu, Nano Lett. 12, 113 (2011).

4. C.-C. Liu, W. Feng, and Y. Yao, Phys. Rev. B 84, 195430 (2011).

5. M. Ezawa, Phys. Rev. Lett. 109, 055502 (2012).

6. M. Ezawa, New J. Phys. 14, 033003 (2012).

7. M. Tahir and U. Schwingenschlsgl, App. Phys. Lett. 101, $132412(2012)$

8. A. Kara, H. Enriquez, A.P. Seitsonen, L.C. Lew Yan Voon, S. Vizzini, B. Aufray, and H. Oughaddou, Surf. Sci. Rep. 67, 1 (2012).

9. A.S. Rodin, A. Carvalho, and A.H. Castro, Neto. Phys. Rev. Lett. 112, 176801 (2014).

10. E.S. Reich, Nature 506, 19 (2014).

11. J. Qiao, Nat. Commun. 5, 4475 (2014).

12. L. Li, Y. Yu, G. J. Ye, Q. Ge, X. Ou, H. Wu, D. Feng, X.H. Chen, and Y. Zhang, Nat. Nanotech. 9, 372 (2014).

13. C.E.P. Villegas and M.R.S. Tavares. Appl. Phys. Lett. 101, 163104 (2012).

14. S. Kling, T. Salger, C. Grossert, and M. Weitz, Phys. Rev. Lett. 107, 240401, (2011).

15. C.E.P. Villegas, M.R.S. Tavares, G.-Q. Hai, and F.M. Peeters. Phys. Rev. B 15, 023015 (2013).

16. W.-F. Tsai, C.-Y. Huang, T.-R. Chang, H. Lin, H.-T. Jeng, and A. Bansil, Nat. Commun. 4,1500 (2013).

17. S. Datta, Electronic Transport in Mesoscopic Systems, (Cambridge University Press, New York, 2007).

18. J. Bird, D. Ferry and S. Goodnick, Transport in Nanostrucutre, (Cambridge University Press, New York, 2009). 